\title{
Investigation of $\beta$-Cryptoxanthin Fatty Acid Ester Compositions in Citrus Fruits Cultivated in Japan
}

\author{
Yusuke Wada $^{1}$, Atsuki Matsubara ${ }^{1}$, Takato Uchikata ${ }^{1}$, Yugo Iwasaki ${ }^{2}$, Satoshi Morimoto ${ }^{3}$, \\ Katsuta Kan $^{4}$, Tetsuya Ookura ${ }^{5}$, Eiichiro Fukusaki ${ }^{1}$, Takeshi Bamba ${ }^{{ }^{*}}$
}

${ }^{1}$ Department of Biotechnology, Graduate School of Engineering, Osaka University, Osaka, Japan; ${ }^{2}$ Graduate School of Bioagricultural Sciences, Nagoya University, Nagoya, Japan; ${ }^{3}$ Food Research Center, Ehime Institute of Industrial Technology, Matsuyama, Japan; ${ }^{4}$ Omishima Fruits Processing Co. Ltd., Imabari, Japan; ${ }^{5}$ Food Function Division, National Food Research Institute, National Agricultural and Food Research Organization, Tsukuba, Japan.

Email: "bamba@bio.eng.osaka-u.ac.jp

Received May $26^{\text {th }}, 2013$; revised June $26^{\text {th }}, 2013$; accepted July $5^{\text {th }}, 2013$

Copyright (C) 2013 Yusuke Wada et al. This is an open access article distributed under the Creative Commons Attribution License, which permits unrestricted use, distribution, and reproduction in any medium, provided the original work is properly cited.

\begin{abstract}
In this study, a catalog of $\beta$-cryptoxanthin $(\beta \mathrm{CX})$ and its fatty acid esters $(\beta \mathrm{CXFAs})$ in citrus fruits were constructed. The peel and pulp of citrus fruits from nine Japanese cultivars and one foreign cultivar were surveyed by supercritical fluid chromatography/triple quadrupole mass spectrometry (SFC/QqQMS). In each citrus sample, the major components were $\beta \mathrm{CX}$ and its laurate ester, myristate ester, palmitate ester, and oleate ester. However, the composition ratio of $\beta C X F A$ varied with the citrus breed and between the pulp and the peel of the fruit in most cultivars. The SFC/QqQMS system could be used to obtain new information about $\beta$ CXs and carry out further discussion on the feature and tendency of each citrus variety.
\end{abstract}

Keywords: $\beta$-Cryptoxanthin Fatty Acid Ester; Supercritical Fluid Chromatography

\section{Introduction}

Carotenoids are fat-soluble pigments that are configured by combining eight isoprene units and are known to have various physiological functions attributed to their antioxidant activity, such as prevention of heart disease and cancer. $\beta$-Carotene, $\beta$-carotene, lycopene, lutein, zeaxanthin, and $\beta$-cryptoxanthin $(\beta \mathrm{CX})$ constitute more than $90 \%$ of the carotenoid content of the human body [1]. Carotenoids are not synthesized endogenously by humans; citrus fruits are the primary dietary source of carotenoids. Citrus unshiu (Unshu mikan), a seedless and easy-peeling citrus species, is the most widely consumed fruit in Japan, and its main carotenoid constituent is $\beta \mathrm{CX}$ [2].

$\beta \mathrm{CX}$ has higher absorbability than other carotenoids; therefore, intake of a small amount of citrus fruits is sufficient for the retention of large amounts of $\beta C X$ in the body [3]. Some epidemiological studies have shown that $\beta \mathrm{CX}$ is the only carotenoid that can reduce the risk of cancer, diabetes, and rheumatism $[1,4,5] . \beta \mathrm{CX}$ is a xanthophyll that exists in free as well as fatty-acid-esterified forms in physiological tissues [6]. $\beta$-Cryptoxanthin fatty

${ }^{*}$ Corresponding author. acid ester ( $\beta$ CXFA) has high hydrophobicity, and it has structurally similar compounds having different types of fatty acid chains. In our previous research, we developed a technique based on supercritical fluid chromatography/ triple quadrupole mass spectrometry (SFC/QqQMS), which has much higher sensitivity and throughput as compared to the conventional liquid chromatographic techniques, for the analysis of $\beta \mathrm{CX}$ and $\beta$ CXFAs [7].

Recently in Japan, numerous $\beta$ CX-rich citrus breeds have been developed for promoting the consumption of domestic citrus varieties. Japanese citrus varieties such as Unshu mikan have higher $\beta C X$ content than the citrus varieties cultivated in the US [8]. For further discussion on the feature and tendency of each Japanese citrus species, the $\beta \mathrm{CX}$ and $\beta$ CXFA profiles must be investigated. Therefore, in this study, a catalog of the $\beta C X$ and $\beta C X F A$ in nine kinds of Japanese citrus fruits was created using the aforementioned SFC/QqQMS system.

\section{Materials and Methods}

\subsection{Samples and Chemicals}

Carbon dioxide (99.99\% purity), which was used as the 
mobile phase in SFC, was purchased from Neriki Gas (Osaka, Japan). Methanol (HPLC grade), which was used as the modifier and make-up solvent, was obtained from Kishida Chemical Co., Ltd. (Osaka, Japan). Ammonium formate, which was used as an additive, was purchased from Sigma-Aldrich Co. (Milwaukee, WI, USA). Acetone (HPLC grade), which was used as the extraction solvent, was purchased from Kishida Chemical Co., Ltd.

$\beta \mathrm{CX}$ was purchased from Wako Pure Chemical Industries, Ltd. (Osaka, Japan). $\beta$ CXFAs such as laurate (C12:0) ester, myristate (C14:0) ester, palmitate (C16:0) ester, stearate $(\mathrm{C} 18: 0)$ ester, oleate $(\mathrm{C} 18: 1)$ ester, linoleate (C18:2) ester, linolenate (C18:3) ester, eicosapentaenoic acid (EPA, C20:5) ester, and docosahexaenoic acid (DHA, C22:6) ester, whose structures are provided in Figure 1, were synthesized [7].

Unshu mikan (C. unshiu), Dekopon $((C$. unshiu $\times C$. sinensis $) \times C$. reticulata), Iyokan $(C$. iyo), Setoka (C. rutaceae), Kiyomi $(C$. unshiu $\times C$. sinensis $)$, Ponkan $(C$. reticulata $)$, Harumi $((C$. unshiu $\times C$. sinensis $) \times C$. reticulata), Harehime (Rutaceae Citrus), and Amakusa (Rutaceae Citrus) were purchased from a market in Omishima (Ehime, Japan) in 2010 (from January to March). Orange (C. sinensis), grown in the US, was purchased from a market in Suita (Osaka, Japan) in January 2012. The breed trees of citrus fruits are shown in Figures 2 (a) and (b).

\subsection{SFC/QqQMS Analysis}

SFC/QqQMS analysis was performed using a Waters

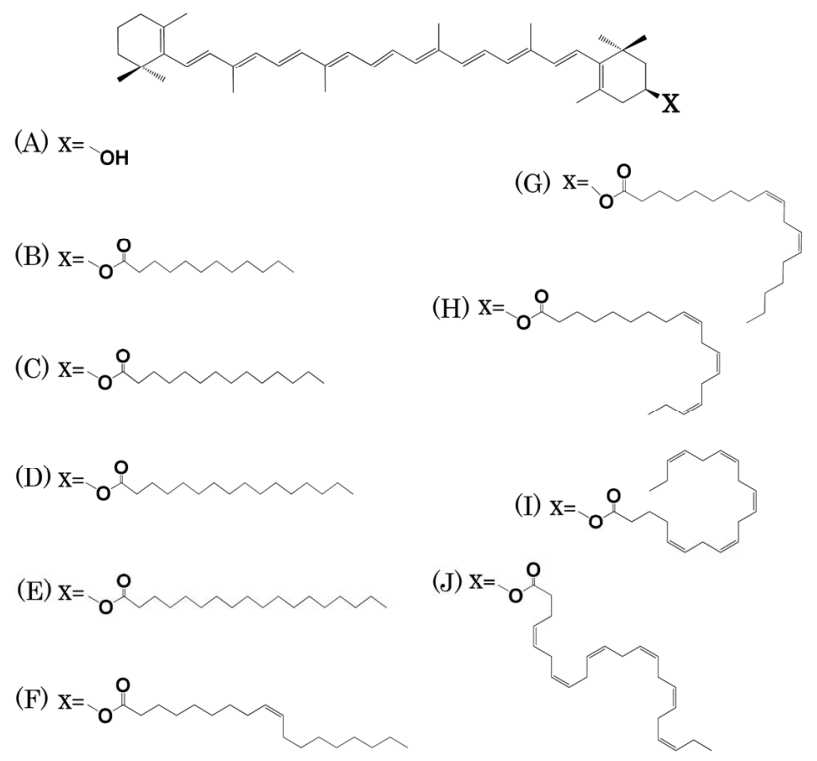

Figure 1. Structures of $\beta C X$ and $\beta C X F A s$ considered in this research. (A) $\beta C X$ (free form) and $\beta C X F A s$ (B) C12:0 ester, (C) C14:0 ester, (D) C16:0 ester, (E) C18:0 ester, (F) C18:1 ester, (G) C18:2 ester, (H) C18:3 ester, (I) C20:5 ester, and (J) C22:6 ester.

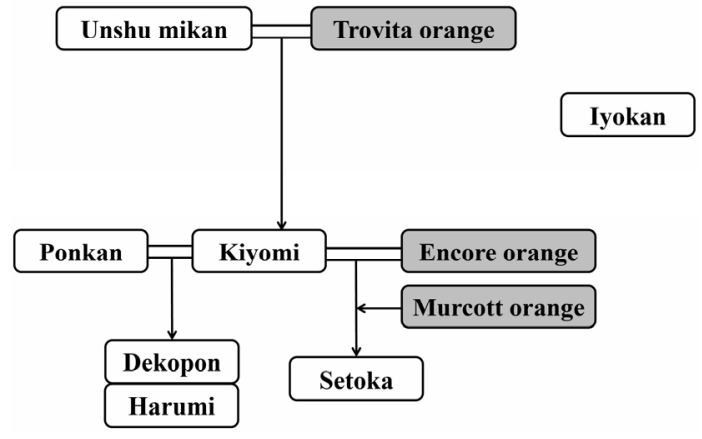

(a)

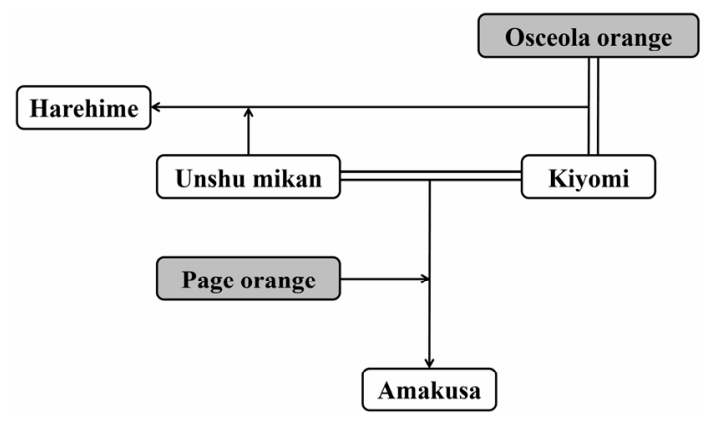

(b)

Figure 2. The breed trees of citrus fruits. The cultivars shaded in gray were not surveyed in this study.

Analytical SFC Method Station equipped with an $\mathrm{AB}$ SCIEX Q TRAP 5500 (AB SCIEX) triple quadrupole mass spectrometer. An intelligent HPLC pump PU-2080 (JASCO Co.) was used to supply the make-up solvent, which enhances ionization. The SFC and QqQMS analysis were controlled by SuperChrom automated control module software and Analyst software (ver. 1.5), respectively.

$\beta \mathrm{CX}$ and $\beta \mathrm{CXFAs}$ were extracted from citrus samples by the following procedure. First, $20 \mathrm{mg}$ of the dried peel was weighed in a glass tube, and $450 \mu \mathrm{L}$ acetone and 50 $\mu \mathrm{L} \beta \mathrm{CX}$ margarate (C17:0) ester (internal standard) were added. Then, ultrasonication was carried out for $10 \mathrm{~min}$. Finally, centrifugation was carried out at 16,000 $g$ for 5 min, and the supernatant was subjected to SFC-QqQMS analysis.

The SFC/QqQMS analysis method was constructed in our previous study [7]. Carbon dioxide and modifier solvent were used as mobile phase, and their flow rate ratio was 9:1 (total flow rate: $3 \mathrm{~mL} / \mathrm{min}$ ). Modifier solvent was methanol containing $0.1 \%(\mathrm{w} / \mathrm{v})$ ammonium formate. This solution was also used as the make-up solvent (flow rate: $0.1 \mathrm{~mL} / \mathrm{min}$ ). The oven temperature and backpressure were set at $35^{\circ} \mathrm{C}$ and $10 \mathrm{MPa}$, respectively. Electrospray ionization (ESI) was used, and detection was performed in the positive-ion mode. The ion source and QqQMS parameters were as follows: curtain gas, 40 psi; collision gas, 7; ion spray voltage, $5500 \mathrm{~V}$; ion source 
gas 1, 60 psi; ion source gas 2, 60 psi; declustering potential, $61 \mathrm{~V}$; entrance potential, $10 \mathrm{~V}$; collision energy, $23 \mathrm{~V}$; collision cell exit potential, $18 \mathrm{~V}$.

\section{Results and Discussion}

\subsection{Analysis of $\beta C X$ and $\beta C X F A s$ in Citrus Peel}

First, $\beta \mathrm{CX}$ and $\beta \mathrm{CXFAs}$ in the peel of citrus fruits from the nine domestic cultivars and the foreign cultivar were analyzed by SFC/QqQMS. The main components of the peel were $\beta \mathrm{CX}$, and $\mathrm{C} 12: 0$ ester, $\mathrm{C} 14: 0$ ester, $\mathrm{C} 16: 0$ ester and $\mathrm{C} 18: 1$ ester. In addition, the $\mathrm{C} 18: 0$ ester was detected in each citrus peel, albeit in trace amounts. Other $\beta \mathrm{CX}$ FAs for which standards are not available, such as C4:0 ester, C6:0 ester, C8:0 ester and C10:0 ester, were also targeted by theoretically estimating the analytical parameters. The structure determination procedure adopted in our previous study was used in the present analysis as well. The results confirmed that citrus peels contain small amounts of C4:0 ester, C6:0 ester, C8:0 ester, and C10:0 ester. However, the amounts of C6:0 ester and C8:0 ester in Orange and those of C4:0 ester, C6:0 ester and C8:0 ester in Amakusa were less than the quantification limit (Table 1). Next, the composition ratios of the components of the studied citrus fruits were compared. This ratio was calculated by dividing the peak area for each component by the total peak area of all the components in the given citrus peel sample. The ratios for each citrus peel are depicted in the form of a pie chart in Figure 3. Unshu mikan had the highest ratios of $\beta \mathrm{CX}$ and C10:0 ester $(49.1 \% \pm 3.4 \%$ and $2.6 \% \pm 0.2 \%$, respectively) among all the citrus cultivars. Harumi, Dekopon, Harehime, and Orange had the highest C12:0 ester, C14:0 ester, C16:0 ester, and C18:1 ester contents, respectively. The $\beta C X F A-t o-t o t a l-\beta C X$ ratio was the high- est in Dekopon. In addition, the concentrations of the major $\beta$ CXFAs in Unshu mikan, Ponkan, Iyokan, Setoka, and Harumi decreased in the following order: C12:0 ester $>$ C14:0 ester $>$ C16:0 ester. Further, the concentrations of these $\beta$ CXFAs in Kiyomi, Dekopon, and Amakusa decreased in the order C12:0 ester $>$ C16:0 ester $>$ C14:0 ester. In contrast, the C16:0 ester was present in the highest concentration in Orange and Harehime, and the concentrations of the abovementioned three esters decreased in the order $\mathrm{C} 16: 0$ ester $>\mathrm{C} 12: 0$ ester $>\mathrm{C} 14: 0$ ester. Thus, the major $\beta$ CXFAs present in all the citrus peel varieties were similar, but their composition ratios varied.

\subsection{Analysis of $\beta C X$ and $\beta C X F A s$ in Citrus Pulp}

The $\beta \mathrm{CX}$ and $\beta \mathrm{CXFA}$ composition in the ten kinds of citrus pulp samples was determined by SFC/QqQMS. The results showed that the compounds detected in the pulp were similar to those in the peel (Table 2). However, the amounts of $\beta$ CXFAs such as C4:0 ester, C6:0 ester and C8:0 ester, were less than the quantification limit in most of the citrus breeds. While C4:0 and C8:0 esters were present in Unshu mikan, Ponkan, and Setoka, the C6:0 ester was present only in Unshu mikan. The relative standard deviation (RSD) of the peak area of the C18:0 ester was large, from $10 \%$ to $98 \%$, owing to the low peak intensity and large error margin in the extraction process. The composition ratio for each citrus pulp sample is shown in Figure 4 as a pie chart. Orange had the highest ratio of $\beta \mathrm{CX} 60.4 \% \pm 0.5 \%$, which implied that this citrus fruit has a much lower $\beta \mathrm{CXFA}$-to-total- $\beta \mathrm{CX}$ ratio than do the domestic varieties. This result contrasted that obtained in the analysis of the citrus peels, according to which Unshu mikan had the highest $\beta C X$ content. The

Table 1. $\beta C X$ and $\beta C X F A$ composition in citrus peel ( $\%$ of total $\beta C X s, n=3)$.

\begin{tabular}{|c|c|c|c|c|c|c|c|c|c|c|}
\hline Citrus varieties & Free form & $\mathrm{C} 4: 0$ ester & C6:0 ester & C8:0 ester & C10:0 ester & C12:0 ester & C14:0 ester & $\mathrm{C} 16: 0$ ester & C18:0 ester & C18:1 ester \\
\hline Unshu mikan & $49.1 \pm 3.4$ & $0.34 \pm 0.01$ & $0.19 \pm 0.00$ & $0.45 \pm 0.03$ & $2.6 \pm 0.2$ & $19.0 \pm 1.4$ & $15.4 \pm 1.1$ & $10.0 \pm 0.7$ & $0.31 \pm 0.02$ & $2.7 \pm 0.2$ \\
\hline Orange & $39.8 \pm 0.1$ & $0.29 \pm 0.04$ & n.q. & n.q. & $0.45 \pm 0.01$ & $15.4 \pm 0.4$ & $11.3 \pm 0.4$ & $17.9 \pm 0.2$ & $0.94 \pm 0.03$ & $14.0 \pm 0.1$ \\
\hline Kiyomi & $20.5 \pm 0.6$ & $0.13 \pm 0.02$ & $0.12 \pm 0.01$ & $0.26 \pm 0.01$ & $1.9 \pm 0.1$ & $28.3 \pm 1.6$ & $19.4 \pm 0.6$ & $20.9 \pm 1.1$ & $1.12 \pm 0.07$ & $7.4 \pm 0.2$ \\
\hline Dekopon & $16.5 \pm 0.4$ & $0.14 \pm 0.05$ & $0.15 \pm 0.04$ & $0.25 \pm 0.02$ & $1.9 \pm 0.1$ & $26.3 \pm 1.3$ & $21.1 \pm 1.5$ & $21.2 \pm 0.4$ & $1.73 \pm 0.17$ & $10.8 \pm 0.4$ \\
\hline Iyokan & $31.3 \pm 0.8$ & $0.22 \pm 0.01$ & $0.16 \pm 0.01$ & $0.15 \pm 0.03$ & $1.1 \pm 0.0$ & $24.5 \pm 0.7$ & $18.4 \pm 0.2$ & $16.1 \pm 1.1$ & $0.34 \pm 0.01$ & $7.7 \pm 0.3$ \\
\hline Setoka & $29.3 \pm 1.0$ & $0.40 \pm 0.02$ & $0.36 \pm 0.01$ & $0.42 \pm 0.01$ & $1.8 \pm 0.1$ & $23.7 \pm 1.0$ & $18.2 \pm 0.5$ & $16.2 \pm 0.1$ & $1.65 \pm 0.04$ & $8.1 \pm 0.0$ \\
\hline Harehime & $28.0 \pm 1.3$ & $0.08 \pm 0.01$ & $0.09 \pm 0.00$ & $0.13 \pm 0.01$ & $1.0 \pm 0.1$ & $18.5 \pm 1.0$ & $16.2 \pm 0.6$ & $24.1 \pm 1.5$ & $1.44 \pm 0.07$ & $10.5 \pm 0.4$ \\
\hline Harumi & $28.2 \pm 0.1$ & $0.23 \pm 0.01$ & $0.22 \pm 0.01$ & $0.30 \pm 0.02$ & $1.6 \pm 0.1$ & $30.3 \pm 0.9$ & $20.6 \pm 0.3$ & $12.2 \pm 0.3$ & $0.31 \pm 0.03$ & $6.0 \pm 0.1$ \\
\hline Amakusa & $29.8 \pm 0.0$ & n.q. & n.q. & n.q. & $1.1 \pm 0.1$ & $21.5 \pm 1.1$ & $16.7 \pm 0.3$ & $21.2 \pm 1.2$ & $0.72 \pm 0.10$ & $9.0 \pm 0.4$ \\
\hline
\end{tabular}



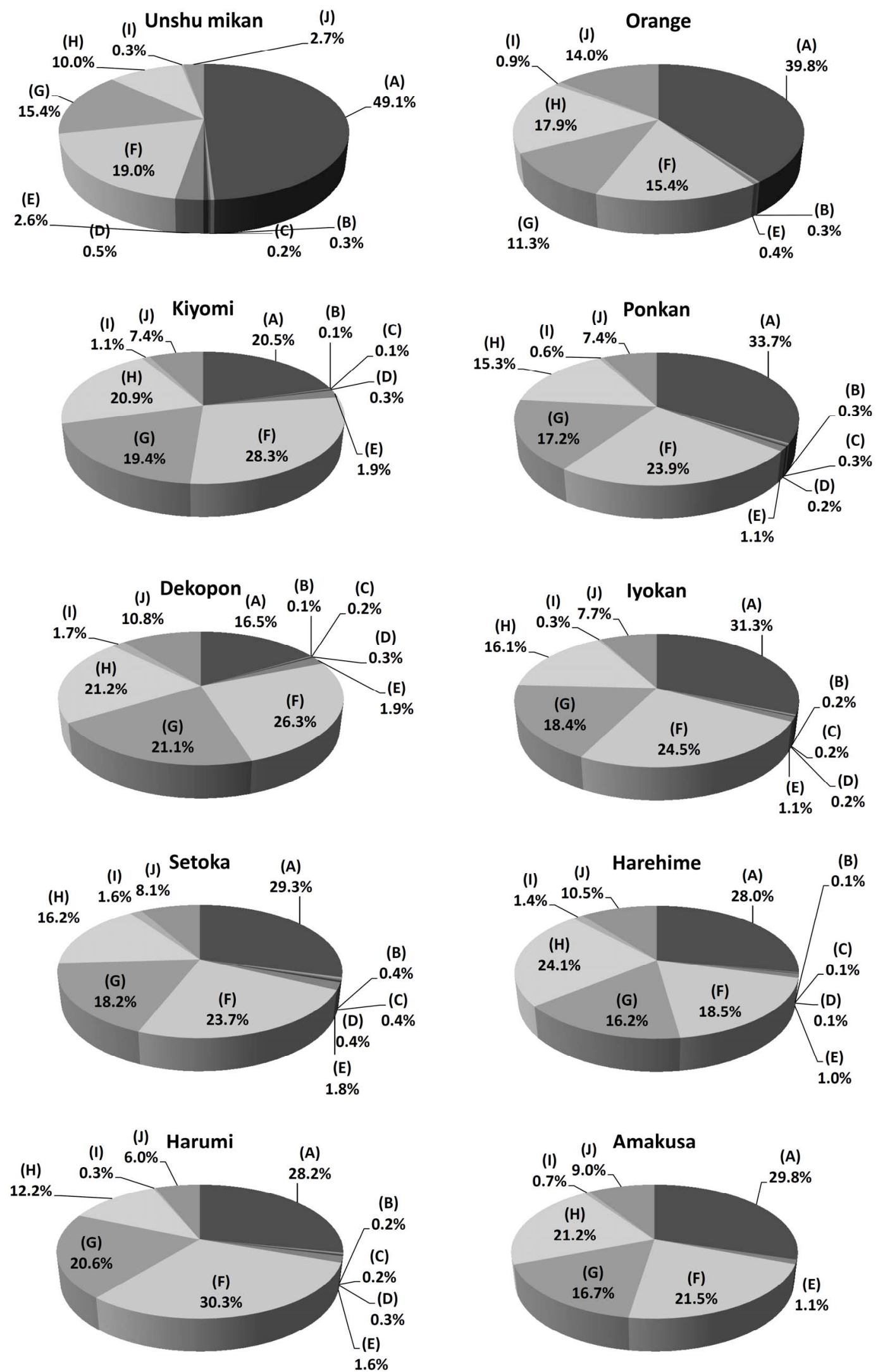

Figure 3. Composition ratio of $\beta C X s$ in citrus peel: (A) Free form, (B) C4:0 ester, (C) C6:0 ester, (D) C8:0 ester, (E) C10:0 ester, (F) C12:0 ester, (G) C14:0 ester, (H) C16:0 ester, (I) C18:0 ester, and (J) C18:1 ester. 

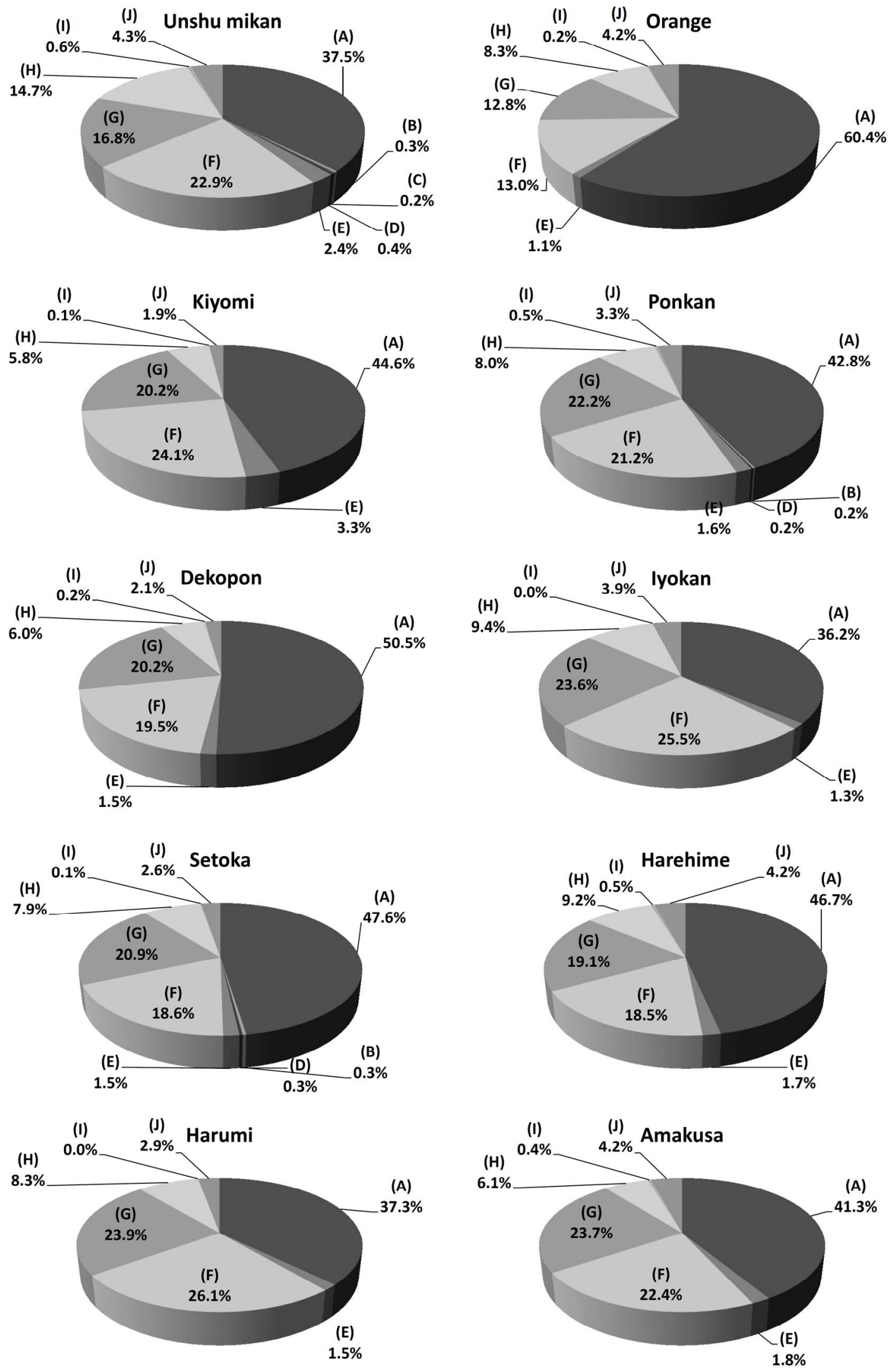

Figure 4. Composition ratio of $\beta C X$ and $\beta C X F A s$ in citrus pulp: (A) Free form $(\beta C X)$ and (B) $C 4: 0$ ester, (C) C6:0 ester, (D) C8:0 ester, (E) C10:0 ester, (F) C12:0 ester, (G) C14:0 ester, (H) C16:0 ester, (I) C18:0 ester, and (J) C18:1 ester. 
Table 2. $\beta C X$ and $\beta C X F A$ composition in citrus pulp $(\%$ of total $\beta C X s, n=3)$.

\begin{tabular}{cccccccccccc}
\hline Citrus varieties & Free form & C4:0 ester & C6:0 ester & C8:0 ester & C10:0 ester & C12:0 ester & C14:0 ester & C16:0 ester & C18:0 ester & C18:1 ester \\
\hline Unshu mikan & $37.5 \pm 2.3$ & $0.28 \pm 0.05$ & $0.21 \pm 0.01$ & $0.39 \pm 0.05$ & $2.4 \pm 0.2$ & $22.9 \pm 1.3$ & $16.8 \pm 0.2$ & $14.7 \pm 1.4$ & $0.58 \pm 0.06$ & $4.3 \pm 0.1$ \\
Orange & $60.4 \pm 0.5$ & n.q. & n.q. & n.q. & $1.1 \pm 0.1$ & $13.0 \pm 0.8$ & $12.8 \pm 0.6$ & $8.3 \pm 1.0$ & $0.20 \pm 0.15$ & $4.2 \pm 0.2$ \\
Kiyomi & $44.6 \pm 1.9$ & n.q. & n.q. & n.q. & $3.3 \pm 0.2$ & $24.1 \pm 1.5$ & $20.2 \pm 1.0$ & $5.8 \pm 0.5$ & $0.06 \pm 0.06$ & $1.9 \pm 0.0$ \\
Ponkan & $42.8 \pm 0.6$ & $0.20 \pm 0.02$ & n.q. & $0.20 \pm 0.03$ & $1.6 \pm 0.0$ & $21.2 \pm 2.2$ & $22.2 \pm 1.1$ & $8.0 \pm 0.6$ & $0.47 \pm 0.05$ & $3.3 \pm 0.2$ \\
Dekopon & $50.5 \pm 1.6$ & n.q. & n.q. & n.q. & $1.5 \pm 0.1$ & $19.5 \pm 1.7$ & $20.2 \pm 1.1$ & $6.0 \pm 0.1$ & $0.18 \pm 0.09$ & $2.1 \pm 0.1$ \\
Iyokan & $36.2 \pm 1.2$ & n.q. & n.q. & n.q. & $1.3 \pm 0.1$ & $25.5 \pm 3.4$ & $23.6 \pm 2.2$ & $9.4 \pm 0.4$ & $0.03 \pm 0.01$ & $3.9 \pm 0.4$ \\
Setoka & $47.6 \pm 1.5$ & $0.34 \pm 0.02$ & n.q. & $0.30 \pm 0.03$ & $1.5 \pm 0.0$ & $18.6 \pm 0.5$ & $20.9 \pm 1.6$ & $7.9 \pm 0.2$ & $0.14 \pm 0.13$ & $2.6 \pm 0.6$ \\
Harehime & $46.7 \pm 1.9$ & n.q. & n.q. & n.q. & $1.7 \pm 0.2$ & $18.5 \pm 1.5$ & $19.1 \pm 1.2$ & $9.2 \pm 1.1$ & $0.53 \pm 0.17$ & $4.2 \pm 0.4$ \\
Harumi & $37.3 \pm 1.6$ & n.q. & n.q. & n.q. & $1.5 \pm 0.1$ & $26.1 \pm 3.2$ & $23.9 \pm 1.8$ & $8.3 \pm 0.4$ & $0.01 \pm 0.01$ & $2.9 \pm 0.1$ \\
Amakusa & $41.3 \pm 2.2$ & n.q. & n.q. & n.q. & $1.8 \pm 0.3$ & $22.4 \pm 2.2$ & $23.7 \pm 1.3$ & $6.1 \pm 0.3$ & $0.44 \pm 0.08$ & $4.2 \pm 0.1$ \\
\hline
\end{tabular}

highest C10:0 ester content was indicated in Kiyomi in the pulp analysis, as opposed to Unshu mikan in the peel analysis. Similarly, pulp analysis indicated the highest C16:0 ester content in Unshu mikan, while peel analysis had indicated the highest C16:0 ester content in Harehime. Furthermore, the citrus species with the highest $\beta$ CXFA-to-total- $\beta$ CX ratio was indicated to be Iyokan by pulp analysis but Dekopon by peel analysis. However, in both pulp and peel analyses, the highest C12:0 ester content was detected in Harumi. These results indicated that the $\beta \mathrm{CX}$ composition differed between the peel and the pulp for most citrus cultivars (Table 3(A)). The concentrations of the major $\beta$ CXFAs in the pulp of Unshu mikan, Orange, Kiyomi, Iyokan, and Harumi decreased in the order C12:0 ester $>\mathrm{C} 14: 0$ ester $>\mathrm{C} 16: 0$ ester. The concentrations of these three esters showed identical trends, both in the peel and in the pulp, in the case of Unshu mikan, Iyokan, and Harumi. The concentrations of the $\beta$ CXFAs in Ponkan, Dekopon, Setoka, Harehime, and Amakusa decreased in the following order: C14:0 ester $>\mathrm{C} 12: 0$ ester $>\mathrm{C} 16: 0$ ester. These results indicated that the composition ratio of the three major $\beta$ CXFAs differed between the peel and the pulp for most citrus cultivars (Table 3(B)).

\section{Conclusion}

In this study, a catalog of the $\beta \mathrm{CXs}$ and $\beta \mathrm{CXFAs}$ presenting in the peel and pulp of ten kinds of citrus species was constructed. In each citrus sample, the major carotenoid components were $\beta \mathrm{CX}$ and $\mathrm{C} 12: 0$ ester, C14:0 ester, C16:0 ester and C18:1 ester. However, the composition ratio of the $\beta \mathrm{CXs}$ in the peel and pulp varied with the citrus breed. The Japanese citrus species had higher $\beta$ CXFA-to-total- $\beta$ CX ratios than the US species did. Because the xanthophyll esterified-form is more bioavailable than its free-form counterpart [9], it was suggested
Table 3. Comparison of $\beta \mathrm{CX}$ composition ratio between peel and pulp. (A) The composition ratio; (B) The ratio of major $\beta$ CXFAs (C12:0 ester, C14:0 ester and C16:0 ester).

(A)

\begin{tabular}{cc}
\hline Similar among peel and pulp & Differ among peel and pulp \\
\hline Harumi & $\begin{array}{c}\text { Unshu mikan, Kiyomi, Ponkan, } \\
\text { Dekopon, Iyokan, Setoka, } \\
\text { Harehime, Amakusa, Orange }\end{array}$ \\
\hline (B) & Differ among peel and pulp \\
\hline Unshu mikan, Iyokan, Harumi & $\begin{array}{c}\text { Kiyomi, Ponkan, Dekopon, Setoka, } \\
\text { Harehime, Amakusa, Orange }\end{array}$ \\
\hline
\end{tabular}

that the Japanese citrus species had higher nutritional value than the US species. The $\beta C X$ composition in the peel and pulp was similar in the case of Unshu mikan, Iyokan, and Harumi but different in other citrus varieties. The use of the SFC/QqQMS system helped in obtaining new information about $\beta \mathrm{CXs}$ in citrus fruits and in carrying out further studies on the feature of each citrus variety. SFC/QqQMS analysis is expected to contribute significantly to research and development activities related to $\beta \mathrm{CXs}$.

\section{Acknowledgements}

This work was partially supported by the Ministry of Agriculture, Forestry and Fisheries of Japan.

\section{REFERENCES}

[1] S. Männistö, S. A. Smith-Warner, D. Spiegelman, D. Albanes, K. Anderson, P. A. Brandt, J. R. Cerhan, G. Colditz, D. Feskanich, J. L. Freudenheim, E. Giovannucci, R. A. Goldbohm, S. Graham, A. B. Miller, T. E. Rohan, J. Virtamo, W. C. Willett and D. J. Hunter, "Dietary Caro- 
tenoids and Risk of Lung Cancer in a Pooled Analysis of Seven Cohort Studies," Cancer Epidemiology Biomarkers \& Prevention, Vol. 13, 2004, pp. 40-48.

[2] K. L. Godner, R. L. Rouseff and H. J. Hofsommer, “Orange, Mandarin, and Hybrid Classification Using Multivariate Statistics Based on Carotenoid Profiles," Journal of Agricultural and Food Chemistry, Vol. 49, No. 3, 2001, pp. 1146-1150. doi:10.1158/1055-9965.EPI-038-3

[3] M. Yano, Y. Ikoma and M. Sugiura, "Recent Progress in $\beta$-Cryptoxanthin Research," Bulletin of the National Institute of Fruit Tree Science, Vol. 4, 2005, pp. 13-28. doi:10.1021/jf000866o

[4] M. Jukka, K. Paul, J. Ritva and R. Antti, "Dietary Antioxidant Intake and Risk of Type 2 Diabetes," Diabetes Care, Vol. 27, No. 2, 2004, pp. 362-366. doi:10.2337/diacare.27.2.362

[5] R. C. James, G. S. Kenneth, A. M. Linda, R. M. Ted and A. C. Lindsey, "Antioxidant Micronutrients and Risk of Rheumatoid Arthritis in a Cohort of Older Women," American Journal of Epidemiology, Vol. 157, No. 4, 2003, pp. 345-354. doi:10.1093/aje/kwf205

[6] T. Wingerath, W. Stahl and H. Sies, " $\beta$-Cryptoxanthin
Selectively Increases in Human Chylomicrons upon Ingestion of Tangerine Concentrate Rich in $\beta$-Cryptoxanthin Esters," Archives of Biochemistry and Biophysics, Vol. 324, No. 2, 1995, pp. 385-390. doi:10.1006/abbi.1995.0052

[7] Y. Wada, A. Matsubara, T. Uchikata, Y. Iwasaki, S. Morimoto, K. Kan, T. Okura, E. Fukusaki and T. Bamba, "Metabolic Profiling of $\beta$-Cryptoxanthin and Its Fatty Acid Esters by Supercritical Fluid Chromatography Coupled with Triple Quadrupole Mass Spectrometry," Journal of Separation Science, Vol. 34, No. 24, 2011, pp. 3546-3552. doi:10.1002/jssc.201100376

[8] M. Yano, M. Kato, Y. Ikoma, A. Kawasaki, Y. Fukazawa, M. Sugiura, H. Matsumoto, Y. Oohara, A. Nagao and K. Ogawa, "Quantitation of Carotenoids in Raw and Processed Fruits in Japan," Food Science and Technology Research, Vol. 11, No. 1, 2005, pp. 13-18. doi:10.3136/fstr.11.13

[9] P. E. Bowen, S. M. Herbst-Espinosa, E. A. Hussain and M. Stacewicz-Sapuntzakis, "Esterification Dose Not Impair Lutein Bioavailability in Humans," Journal of Nutrition, Vol. 132, 2002, pp. 3668-3673. 